\title{
ON THE INFORMATIONAL CONTENT OF WAGE OFFERS*
}

\author{
By Mehmet BAC ${ }^{1}$
}

Bilkent University, Turkey and Sabanci University, Turkey

\begin{abstract}
This article investigates signaling and screening roles of wage offers in a single-play matching model with two-sided unobservable characteristics. It generates the following predictions as matching equilibrium outcomes: (i) "good" jobs offer premia if "high-quality" worker population is large; (ii) "bad" jobs pay compensating differentials if the proportion of "good" jobs to "low-quality" workers is large; (iii) all firms may offer a pooling wage in markets dominated by "high-quality" workers and firms; or (iv) Gresham's Law prevails: "good" types withdraw if "bad" types dominate the population. The screening/signaling motive thus has the potential of explaining a variety of wage patterns.
\end{abstract}

\section{INTRODUCTION}

Appropriate job-worker matching occurs to the extent that the parties' relevant characteristics are common knowledge, that is, if the existing economic institutions allow for full screening and signaling before the transaction takes place. Interviews and tests are screening devices, whereas the verifiable items in an applicant's CV are signals. Firms can also signal job characteristics through ads, size, employees, and reputation. Despite these instruments, in practice a firm's information about jobrelevant characteristics of its applicants is never perfect, and a worker is never certain as to the attributes of the jobs he/she is offered. The matching problem is therefore a potential source of economy-wide inefficiency.

This article concentrates on one instrument, the offer wage, and investigates its impact on matching efficiency. What signaling and/or screening functions can wage offers perform when nonwage instruments are not available or only partially succeed in transmitting the relevant information $?^{2}$ This question is addressed in a matching model of a large job market populated by observationally indistinguishable, heterogeneous firms and workers. Heterogeneity is introduced in the simplest way, by assuming two basic types of firms and workers, where one type has an advantage

\footnotetext{
* Manuscript received June 1999; revised December 1999.

${ }^{1}$ I would like to thank two anonymous referees for comments and suggestions. The article also benefited from a presentation at the ASSET-95 conference, Istanbul, Turkey. Please address correspondence to: Mehmet Bac, Sabanci University, Faculty of Arts and Sciences, Orhanli, Tuzla, 81474, Istanbul, Turkey. Fax: 90-216-483 9005. E-mail: mbac@sabanciuniv.edu.tr. Fax: 90-3122664948. Email: bac@sabanciuniv.edu.

${ }^{2}$ There is evidence suggesting that wage offers have informational content. See, for instance, Holtzer et al. (1991).
} 
over the other: firms prefer good-quality workers and workers prefer firms with better attributes, wages being equal. However, good-quality workers are much more productive in firms with better attributes; hence, efficiency requires firms and workers of the same type be matched. The approach in this article differs in that the process of matching is explicitly modeled as a noncooperative game where firms offer wages and workers respond with their application decisions. The matching probability at a given wage offer is obtained endogenously, as a feature of the equilibrium outcome. ${ }^{3}$

This simple model generates a rich class of predictions in the form of matching equilibria, relating wage offers and matching efficiency to the distribution of unobservable characteristics: if the proportion of "good" firms to "bad" workers is large, perfect matching occurs through wage offers that do both signaling and screening. In another equilibrium, wages signal firm types but do only partial screening if the good-worker population is sufficiently large. Both firm types offer the same wage in equilibrium if the market is predominantly populated by good workers and good firms. Other equilibria exhibit Gresham's Law in the job market: pessimistic workers and firms of the good type withdraw and take their outside options. Because search is assumed to be costless, any inefficiency of the matching equilibrium outcome is due solely to the two-sided information problem.

Below, I briefly relate the article's predictions to the literature and relegate a more detailed discussion to Section 4. The wage determination literature provides a number of theories explaining observed wage patterns, sources of inter- and intraindustry wage differentials, sizes of compensating differentials and instances where they are paid, and why wages may exceed workers' opportunity costs. The implications derived in this article complement existing explanations for the above phenomena, some of which are termed "anomalies." 4 Because the model is a one-shot matching game, these implications should be relevant especially in the short run.

Efficiency wage and agency models of employment relationships show that paying more than the apparent going wage may deliver a net productivity gain. ${ }^{5}$ Such a strategy can also sort workers into firms that have differential observed compensations. ${ }^{6}$ The explanation in this article is based on unobservable characteristics: the motive of signaling unobserved firm attributes alone can generate a wage differential. This equilibrium outcome arises if the population of good-quality workers is relatively large, that is, if a desirable firm attribute can be signaled through a wage differential at a reasonable cost. The signaling motive is a plausible explanation for many observed intraindustry wage differentials, such as the substantial annual wage

\footnotetext{
${ }^{3}$ The standard approach to modeling matching in labor markets (see Masters, 1999; Coles, 1999 and the references therein) is to postulate an exogenous random matching process.

${ }^{4}$ See Thaler (1989), who reports interesting real-life examples of substantially different wage offers for similar jobs and discusses the theoretical explanations for interindustry wage differentials.

${ }^{5}$ See, for instance, Lazear (1979) and Shapiro and Stiglitz (1984) for theories generating these predictions, and Gibbons and Murphy (1992) and Leonard (1990) for their tests.

${ }^{6}$ The classic article by Roy (1951) espouses this view. Examples of more recent treatments are Cain (1976) and Bulow and Summers (1986).
} 
differential an MBA graduate may receive from two similar jobs in the same city (which is reported in Thaler, 1989).

Compensating differentials play an important role in wage determination when jobs differ with respect to observable attributes. The theory (see Rosen, 1986) stipulates premia should be paid according to the perceived difficulty of the job, but there seems no reason why such premia should be paid when workers and jobs are observationally indistinguishable. I show that the prediction of the theory of compensating differentials continues to hold under two-sided incomplete information provided that the market is largely populated by "good" jobs and "bad" workers. A matching equilibrium exists in which wage offers perform full signaling and screening: jobs with undesirable attributes pay a premium, matching "good" firms and workers at a lower wage than "bad" firms and workers. ${ }^{7}$ This is the only outcome that efficient labor allocation obtains in the present model. The predictions summarized above suggest that the motive of signaling job attributes or screening for worker characteristics through wage offers has the potential of explaining a variety of short-run wage patterns in markets where agents' characteristics are not fully observable.

The article is organized as follows: The next section describes the matching model and its equilibrium concept. Section 3 presents the equilibrium outcomes and Section 4 provides a summary and discussion of results. All proofs are gathered in the Appendix.

\section{THE MODEL}

Consider a sector of the economy with large populations of firms and workers, of measure $M$ and $N$, respectively. Each worker has one unit of indivisible labor for sale, and each firm seeks to buy one unit of labor. There are two possible types of firms $(H$ and $L)$ and workers $(h$ and $l)$. A measure $\rho M((1-\rho) M)$ of firms are of type $H(L)$, and a measure $\pi N((1-\pi) N)$ of workers are of type $h(l)$. Though $\pi$ and $\rho$ are common knowledge, types are privately known.

All firms have the same reservation profit normalized to zero. The profit of a type- $j$ firm paying the wage $w$ to a type- $i$ worker is $R_{j}(i)-w$. I assume that $h$-workers have desirable general abilities that make them more productive than $l$-workers in both types of firms: $R_{H}(h)>R_{H}(l)$ and $R_{L}(h)>R_{L}(l)$. Furthermore, $h$-workers are much more productive in $H$-firms: $R_{H}(h)>R_{L}(h)$. To exemplify, $H$-jobs may be providing better working conditions or be more flexible, which may considerably increase $h$-workers' productivity. $L$-jobs may involve rather routine tasks where general abilities matter less, which would make $l$-workers more productive in $L$-jobs than $H$-jobs: $R_{L}(l)>R_{H}(l)$. Thus, the following ranking of productivities is assumed:

\footnotetext{
${ }^{7}$ See Bac (2000) for a different, multiperiod model in which the firm has monopsony power and "good" workers (temporarily) accept wages lower than their outside options and signal their types on the job.
} 


$$
R_{H}(l)<R_{L}(l)<R_{L}(h)<R_{H}(h)
$$

The utility function of a type- $i$ worker who works in a type- $j$ firm is denoted $u_{i}(w, j)$ and is strictly increasing in $w$. All workers have the same reservation utility $\bar{u},{ }^{8}$ with matching preferences similar to (A1): given the wage $w$, all workers prefer employment in $H$-firms; that is, $u_{i}(w, H)>u_{i}(w, L), i=l, h$. Furthermore, $h$ workers perceive a great difference between the two job attributes. For example, the routine tasks or bad working conditions of $L$-firms may have a more frustrating effect on creative and high-ability workers; that is, for a given wage, $u_{h}(w, L)<u_{l}(w, L)$. Therefore, under complete information $h$-workers must be paid a higher wage than $l$-workers to accept an $L$-job. Conversely, the good attributes of $H$-jobs would suit $h$-workers much better than $l$-workers, and given the wage $w$, $h$-workers would derive a greater utility from employment in $H$-firms: $u_{h}(w, H)>u_{l}(w, H)$. Therefore, given that their reservation utility is the same, $h$ workers would accept working in $H$-firms for a lower wage than $l$-workers. To combine these assumptions, for any wage $w,{ }^{9}$

$$
u_{h}(w, L)<u_{l}(w, L)<u_{l}(w, H)<u_{h}(w, H)
$$

The technology and preference assumptions (A1) and (A2) generate a rich class of equilibria in the matching game described below. Using (A2), four minimal wage levels can be defined through the following equalities:

$$
u_{h}\left(\bar{w}_{h}, L\right)=u_{h}\left(\underline{w}_{h}, H\right)=u_{l}\left(\bar{w}_{l}, L\right)=u_{l}\left(\underline{w}_{l}, H\right)=\bar{u}
$$

The wage $\bar{w}_{i}$ makes type- $i$ workers indifferent between working in $L$-firms and taking their outside option. Similarly, the minimum wage that a type- $i$ worker would accept from an $H$-firm is $\underline{w}_{i}$. Since both worker types prefer $H$-jobs, $\bar{w}_{i}>\underline{w}_{i}$; that is, under complete information a compensating wage differential is required to have the type- $i$ worker accept the $L$-job instead of the $H$-job. By (A2), this compensation should be relatively large for $h$-workers.

Finally, I make a simplifying assumption according to which there are gains from matching between firms and workers of the same type, but for $i \neq j$, the total surplus from a $j-i$ matching is negative:

$$
R_{H}(h)>\underline{w}_{h}, \quad R_{L}(l)>\bar{w}_{l} \quad \text { and } \quad R_{H}(l)<\underline{w}_{l}, \quad R_{L}(h)<\bar{w}_{h}
$$

Thus, incomplete information may have serious inefficiency consequences because $l$-workers would like to convince firms that they are of type $h$, while $L$-firms will try to conceal their types in order to attract $h$-workers. Assumption (A3) implies that

\footnotetext{
${ }^{8}$ Though this is a strong assumption (because workers with better general abilities may have better outside options), it is qualitatively inconsequential to our results provided that the surplus from an $H-h$ matching remains positive and $h$-workers' reservation utility is not too high relative to $l$-workers.

${ }^{9}$ Such interpersonal utility comparisons are inevitable in this context. Firms must know how exactly the two worker types trade off wages for job attributes. For instance, a firm posting a wage offer has to form expectations about the types of its prospective applicants, the lowest wage that would signal an $H$-firm and be rejected by $l$-workers but accepted by $h$-workers, whether a wage offer would signal no information and be accepted by both types of workers, and so on.
} 
$h$-workers would prefer taking their outside options if $H$-firms withdraw from the market, and similarly, that it is optimal for $H$-firms to shut down if only $l$-workers are seeking jobs. ${ }^{10}$ It is also immediately evident that the usual "single crossing property" (commonly assumed in signaling models) does not apply here. Only one instrument is available for conveying type information: offer wages for the firms and acceptance decisions for the workers. These features stem from my objective to focus exclusively on the informational role of wage offers, their signaling and screening functions in a matching model.

The job market operates through the following stages: It opens with simultaneous wage announcements by the firms. The strategy of firm $m$ of type $j$ is to post one vacancy and a wage $w_{j}^{m} \geq 0,{ }^{11}$ which remains fixed during the matching process. On the basis of these offers, workers revise their beliefs about the types of firms. A system of beliefs generated by these offers is denoted $\{\hat{\rho}\}$, mapping each possible wage offer into the interval $[0,1]$. A type- $i$ worker's decision problem consists of determining an acceptance list $\kappa_{i}$ that ranks the firms according to the expected utilities corresponding to their offers. The firm offering the highest expected utility is placed on top, followed by the second-best offer and so on. All offers that yield an expected utility less than $\bar{u}$ are rejected, and those yielding the same expected utility are successively but randomly ranked. With their acceptance list in hand, workers meet firms, starting from their first-best choice. This process is assumed to be costless. ${ }^{12}$ A system of beliefs about the types of applicants is denoted $\{\hat{\pi}\}$, mapping the set of all possible offers that receive an application into the interval [0, 1]. If two or more workers apply simultaneously to the same job, the firm randomly chooses one and the couple withdraws from the market. Workers who have not been able to meet, or if they meet, not been chosen by, their first-best choice, continue to search according to their acceptance lists. If a worker exhausts his list he remains unemployed and receives $\bar{u}$. A firm that meets no applicants shuts down. ${ }^{13}$

The expected profit of a type-j firm can be written as

$$
v_{j}=\alpha_{j}\left[\hat{\pi} R_{j}(h)+(1-\hat{\pi}) R_{j}(l)-w_{j}\right]
$$

where $\alpha_{j}$ denotes the probability that the offer $w_{j}$ attracts at least one applicant and $\hat{\pi}$ is the revised probability that the worker (selected among the applicants) is

\footnotetext{
${ }^{10}$ Proposition 6 describes such an equilibrium outcome.

${ }^{11}$ The superscript $m$ will be dropped when all type-j firms make the same offer.

12 This, of course, is a simplification. A side benefit of the costless search assumption is that it leaves the two-sided information problem as the sole source of equilibrium market inefficiency, if any.

${ }^{13}$ Weiss (1990, pp. 35-41) describes a similar matching process with identical firms and heterogeneous workers: each firm announces a wage and a number of jobs. Firms choose randomly among applicants if the number of applicants exceeds posted jobs. However, workers in Weiss' model can make only one application; hence, weigh wages against acceptance probabilities.
} 
of type $h$. I assume that if a measure $f$ of firms make an offer that attracts a measure $g$ of workers, each of these $f$-firms meets a $g$-worker with probability $\min \{1, g / f\} .^{14}$

The strategies and systems of beliefs $\left(\left\{w_{j}^{*}\right\},\left\{\kappa_{i}^{*}\right\},\{\hat{\pi}\},\{\hat{\rho}\}\right)$ must constitute a matching equilibrium, essentially a perfect Bayesian equilibrium with two rather natural restrictions on belief systems (see the Appendix for their formal statements).

The first condition is in the spirit of the Cho-Kreps (1987) Intuitive Criterion. If there is an out-of-equilibrium wage offer $w_{0}$ that a type- $i$ firm would never make, while the other type $j$ would benefit if it so convinces the workers that this offer comes from a $j$-firm, then the workers must put probability zero on type $i$ when they receive the offer $w_{0}$. This condition rules out equilibria in which all firms make the same offer, supported by beliefs " $\hat{\rho}<1$ for $w \in\left(R_{L}(h), R_{H}(h)\right]$ " because such an offer can only come from an $H$-firm.

The second condition is that workers' beliefs should not stop an individual firm bidding up the wage if it is in its own interest to do so. This condition allows for Bertrand-type competition and will have bite whenever equilibria involve $\alpha<1$, that is, whenever firms expect meeting a worker with probability less than one. With beliefs unchanged at the right neighborhood of an equilibrium offer, workers will place the deviant offer $w_{j}+\epsilon$ above $w_{j}$ in their acceptance lists; hence, this firm can attract a larger number of applicants. Note that the firm deviating to a slightly higher offer cannot expect to attract workers with better (unobservable) qualities because higher offers would be accepted by both worker types, which should leave the firm's beliefs about its applicants unchanged. Except in the range $\left[\underline{w}_{h}, \underline{w}_{l}\right)$, any offer accepted by $h$-workers is also accepted by $l$-workers, and no wage lower than $\underline{w}_{h}$ is accepted by any worker. Thus, a firm posting the deviant (out-of-equilibrium) wage $w_{0} \in\left[\underline{w}_{h}, \underline{w}_{l}\right)$ must be convinced that any applicant is of type $h$.

For the rest of the article equilibrium refers to a perfect Bayesian equilibrium that survives these conditions. As the workers' strategies will be rather transparent, hence as the firms' process of updating their beliefs will be relatively straightforward, I will suppress $\left\{\kappa_{i}^{*}\right\}$ and $\{\hat{\pi}\}$ in describing equilibrium strategies for conciseness. Equilibria in which wage offers are devoid of type information are called pooling equilibria, as opposed to separating equilibria where wage offers are clear-cut signals of firm types. Equilibria can also be classified according to the informational content of workers' acceptance strategies, as shown in Table 1: screening for equilibria inducing different, and nonscreening for equilibria inducing identical, acceptance choices. Note that an outcome where one worker type withdraws from the market while the other type accepts some offers is also a screening outcome. In addition to the four possible combinations of types of equilibria, there may be hybrid, or semi-screening, equilibria in which differential acceptance decisions convey no information to one firm

\footnotetext{
14 This and several other assumptions in the article can be motivated by assuming a continuum of firms and workers, and will hold approximately for large populations. There are potential measuretheoretic problems in models with a continuum of agents, focusing on the behavior of subsets (coalitions) of agents. See Hammond et al. (1989), for example. I follow Wolinsky (1990) in taking several features as model primitives instead of going into the terse exercise of deriving them from a model of continuum of agents.
} 
TABLE 1

CLASSIFICATION OF POTENTIAL MATCHING EQUILIBRIA

\begin{tabular}{cccc}
\hline & $\kappa_{l}^{*}=\kappa_{h}^{*}$ & $\kappa_{l}^{*} \neq \kappa_{h}^{*}$ & $\kappa_{l}^{*} \neq \kappa_{h}^{*}$ \\
\hline$w_{H}^{*}=w_{L}^{*}$ & Pooling/nonscreening & Pooling/screening & - \\
$w_{\mathrm{H}}^{*} \neq w_{\mathrm{L}}^{*}$ & - & Separating/screening & Separating/semiscreening \\
\hline
\end{tabular}

type whereas the other firm type is able to predict accurately the type of its applicants. This can happen only if the firms' equilibrium offers are "separating," which explains the empty cell at the top right of Table 1. A separating/nonscreening equilibrium is also impossible because if the two firm types offer different wages, thus separate, $l$-workers and $h$-workers cannot all be indifferent between the two offers (whereas they should, in a nonscreening equilibrium where acceptance choices reveal no type information). This explains the empty cell at the bottom left of Table 1 .

I present below the equilibrium outcome under complete information (fully observable characteristics). Assumption (A3) implies that only $i-i$ matchings will occur; therefore, in this benchmark case the market can be treated as consisting of two submarkets. The Nash equilibrium of this game reproduces the competitive equilibrium outcome.

Proposition 1. A unique equilibrium exists under complete information. $H$-firms offer $w_{H}^{*}=\underline{w}_{h}$ if $\pi N \geq \rho M$, and $w_{H}^{*}=R_{H}(h)$ if $\pi N<\rho M$; these offers are accepted by h-workers. L-firms offer $w_{L}^{*}=\bar{w}_{l}$ if $(1-\pi) N \geq(1-\rho) M$, and $w_{L}^{*}=R_{L}(l)$ if $(1-\pi) N<(1-\rho) M$; these offers are accepted by l-workers.

Consider the case $\pi N \geq \rho M$, which means that $h$-worker population exceeds $H$-firm population. If $w>\underline{w}_{h}$ were an equilibrium offer, it would be accepted by all $h$-workers but a measure $\pi N-\rho M$ would nevertheless be unemployed. Anticipating this, any $H$-firm could deviate to the offer $\underline{w}_{h}$, meet at least one worker with probability one, and decrease its wage costs. This yields $\underline{w}_{h}$ as type- $H$ firms' unique equilibrium wage offer. Similar arguments can be used to show that $w_{H}^{*}=R_{H}(h)$ if $\pi N<\rho M$. Hence, efficient matching is obtained under complete information and all the surplus in equilibrium goes to agents belonging to the relatively smaller population.

\section{UNOBSERVABLE CHARACTERISTICS ON BOTH SIDES}

This section studies the matching game presented in Section 2 under incomplete information. Throughout the analysis I assume $N \geq M$; that is, worker population is larger than firm population. ${ }^{15}$ Let $N(H)=\min \{\rho M, \pi N\}$ and $N(L)=$ $\min \{(1-\rho) M,(1-\pi) N\}$ represent employment in $H$ - and $L$-sectors under efficient matching. The maximum social surplus is

\footnotetext{
15 The analysis of the opposite case does not present any additional difficulty. I focus on the case $N \geq M$ for conciseness of exposition and because I consider it to be more representative of most realworld situations.
} 


$$
Z^{*}=\left[R_{H}(h)-\underline{w}_{h}\right] N(H)+\left[R_{L}(l)-\bar{w}_{l}\right] N(L)
$$

consisting of the surpluses from $H-h$ and $L-l$ matching. The corresponding level of aggregate unemployment is $U^{*}=N-N(H)-N(L)$. The market outcome will be inefficient whenever the actual equilibrium surplus, denoted $Z_{E}$, is below $Z^{*}$. This happens if (i) some types withdraw from the market and/or (ii) matching of opposite types occurs. The measure of inefficiency is therefore $C_{E}=Z^{*}-Z_{E}$.

A few remarks on equilibrium matching outcomes and wage determination may be useful at this stage. Relative proportions of $H$ - to $L$-firms and $h$ - to $l$-workers play an important role in determining the type of equilibrium and matching. For instance, a pooling wage offer is likely in markets populated predominantly by $H$-firms and $h$-workers. The probability of an $H-l$ or $L-h$ matching being low, $H$-firms will not find it beneficial to separate from $L$-firms that imitate them. Given the equilibrium type, wages are determined by demand and supply considerations, that is, the firms' probability of receiving at least one applicant (which depends on the proportion of workers applying to their offer) and the expected type of the applicants. Bidding up the wage slightly increases the number but cannot improve the expected quality of applicants. Bidding down the wage may be beneficial if the firm does not expect a sharp fall in the number of applicants, which depends on whether there is unemployment at the actual wage and how the workers interpret a lower wage.

3.1. Pooling/Nonscreening (PN) Equilibria. I consider first equilibria in which wages and acceptance decisions convey no type information. The set of type distributions $\{\pi, \rho\}$ for which a PN equilibrium exists is shown in Figure 1 by the shaded area. A PN equilibrium exists in markets populated predominately by $H$-firms and $h$-workers. Because $N \geq M$ and all firms offer the same wage $w_{P}^{*}$ accepted by all workers, there will be unemployment, which bids down the pooling wage until $E u_{i}\left(\rho, w_{P}^{*}\right)=\bar{u}$ for $i=l$ and/or $h$. Thus, this pooling offer yields all workers an expected utility equal to their outside surplus. In Figure 1, the area to the northeast of the intersection of the schedules $\pi_{H}\left(w_{P}\right)$ and $\pi_{L}\left(w_{P}\right)$ represents the set $S_{\mathrm{PN}}$ of type distributions such that all firms at least break even by offering the equilibrium wage. ${ }^{16}$ The firms have no incentive to bid up the wage because they are already matched with at least one applicant and bidding up the wage does not improve the quality of applicants. On the other hand, no firm has an incentive to decrease its offer, for it would be interpreted as an $L$-firm. One final condition that remains to be checked is that because there is unemployment, an $L$-firm may consider offering a lower wage, signal its type, and attract only $l$-workers. This will not happen, because the condition $\rho>\bar{\rho}$ implies $w_{P}^{*}<\bar{w}_{l}$ : PN equilibrium wage is lower than the lowest wage that $l$-workers would accept for employment in $L$-firms.

\footnotetext{
${ }^{16} S_{\mathrm{PN}}$ consists of high $\rho$ and $\pi$ values because a high $\rho$ (meaning the proportion of $H$-firms is large) allows for a lower acceptable pooling wage offer $\left(w_{P}^{*}\right.$ is decreasing in $\rho$ ) while a high $\pi$ (meaning the proportion of $h$-workers is large) implies that the firms can earn nonnegative expected profits by attracting both types of workers.
} 


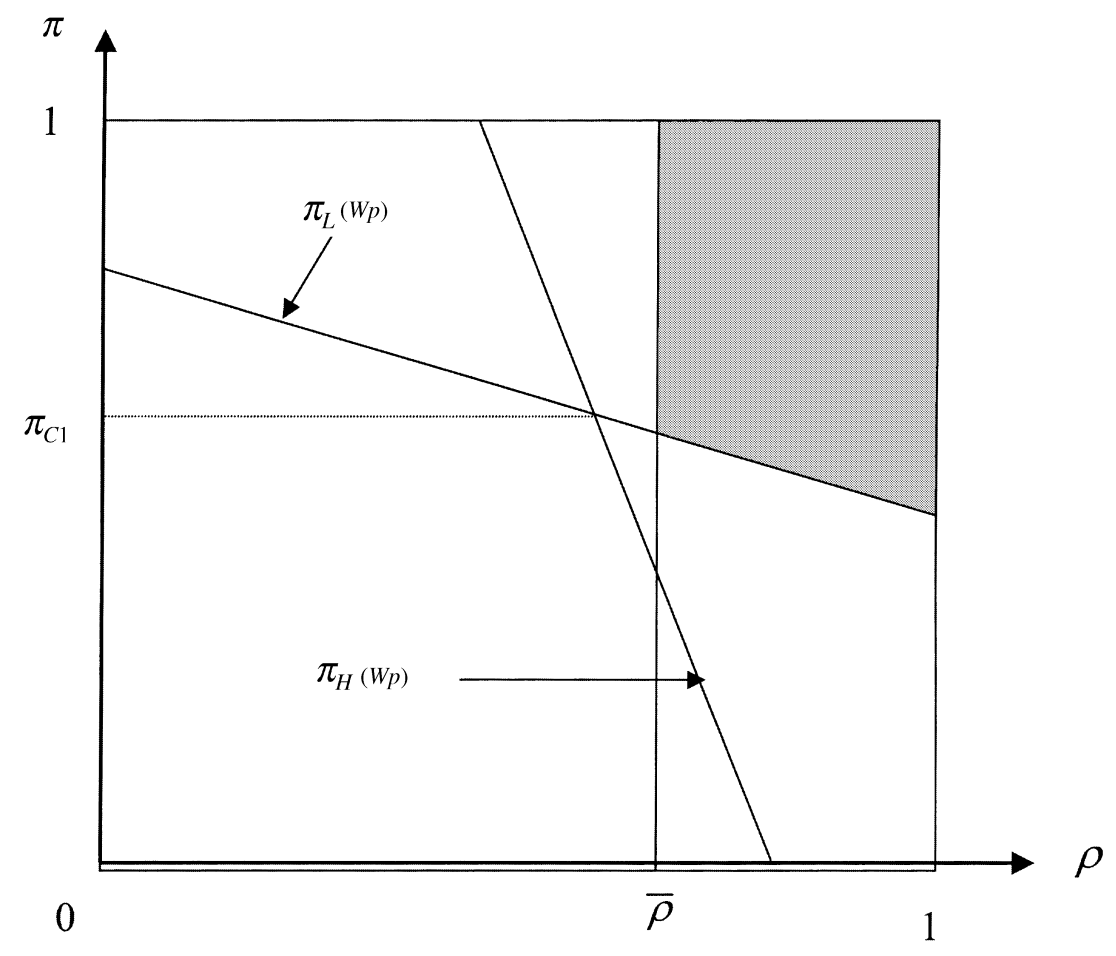

FIGURE 1

RANGE OF POOLING/NONSCREENING EQUILIBRIA AS A FUNCTION OF FIRM AND WORKER POPULATION DISTRIBUTIONS

Proposition 2. If $\{\pi, \rho\} \in S_{\mathrm{PN}}$ and $\rho>\bar{\rho}$, a PN equilibrium exists where all firms offer the same wage $w_{P}^{*}$, which all workers accept. Both $L-h$ and $H-l$ matchings occur; thus, a PN equilibrium displays all types of matching inefficiency.

No wage dispersion is observed in this equilibrium. A single wage clears the market populated by two different types of workers and firms. Though all jobs are filled and unemployment $U^{*}=N-M$ is minimal, the PN equilibrium outcome displays both types of matching inefficiency: a measure $(1-\pi) \rho M$ of $H$-firms are matched with $l$-workers and a measure $\pi(1-\rho) M$ of $L$-firms are matched with $h$-workers. For instance, in the case $\rho M<\pi N$ and $(1-\rho) M<(1-\pi) N$ (H- and $L$-firm populations are smaller than the corresponding worker populations), the measure of inefficiency will be

$$
C_{\mathrm{PN}}=(1-\pi) \rho M\left[R_{H}(h)-\underline{w}_{h}+\underline{w}_{l}-R_{H}(l)\right]+\pi(1-\rho) M\left[R_{L}(l)-\bar{w}_{l}+\bar{w}_{h}-R_{L}(h)\right]
$$

which reflects the surplus that can be generated by dissolving matching of opposite types and constructing the maximum number of proper matching. Inefficiency of PN equilibrium vanishes as $\pi \rightarrow 1$ and $\rho \rightarrow 1$, that is, as firm and worker population distributions homogenize toward the high-quality type. 


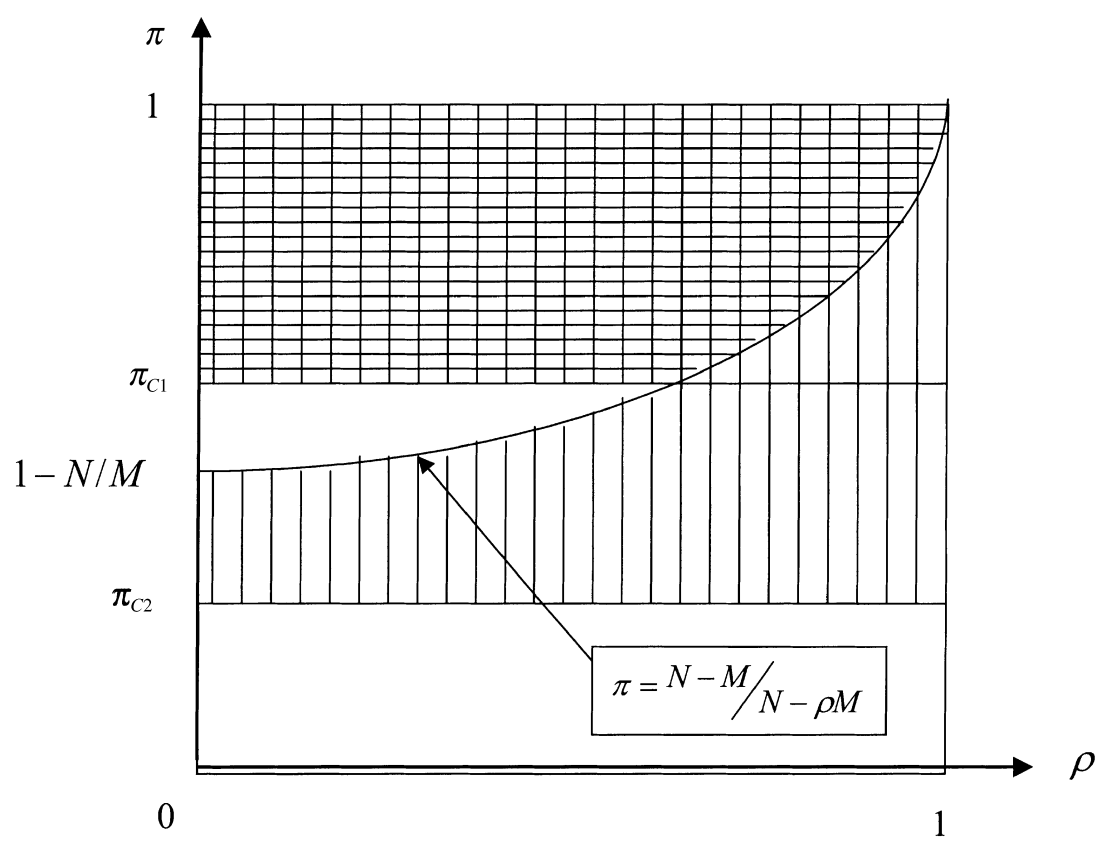

FIGURE 2

RANGE OF SEPARATING/SEMISCREENING EQUILIBRIA AS A FUNCTION OF FIRM AND WORKER POPULATION DISTRIBUTIONS

3.2. Separating/SemiScreening (SSS) Equilibria. An SSS equilibrium involves a pair of distinct wage offers, one for each firm type. All workers accept the higher wage offer and place it at the top of their acceptance lists; therefore, the higher wage offer does no screening. By Assumption (A.3), $L$-firms cannot make a separating wage offer accepted by all workers; therefore, the higher wage offer comes from $H$-firms. $L$-firms make the low offer accepted by $l$-workers only (though ranked below the high offer of $H$-firms). Since $N \geq M, H$-firms cannot obviously hire the entire worker population. Among those who have not been able to match with an $H$-firm, (residual) workers of type- $l$ apply to $L$-firms while (residual) $h$-workers withdraw from the market. Two cases arise according to whether the size of $L$-firms exceeds or not the size of these residual $l$-workers. In the affirmative, the wage in the $L$-sector is $R_{L}(l)$; otherwise it is $\bar{w}_{l}$. $H$-firms' offer is "separating" and higher, but equal to the lowest offer that prevents $L$-firms' imitation. $H$-firms take the risk of being matched with $l$-workers because $h$-worker population (or $\pi$ ) is large enough (Figure 2 ).

Proposition 3. (i) If $\pi \geq \max \left\{\pi_{C 1},(N-M) /(N-\rho M)\right\},{ }^{17}$ the following strategies form an SSS equilibrium: H-firms offer $w_{H}^{*}=\pi R_{L}(h)+(1-\pi) R_{L}(l)$ and

${ }^{17}$ The two critical beliefs $\pi_{C 1}$ and $\pi_{C 2}$ are defined in the Appendix. 
L-firms offer $w_{L}^{*}=R_{L}(l)$. Any wage $w \geq w_{H}^{*}$ is interpreted as coming from an H-firm; other wages are interpreted as an L-firm's offer.

(ii) If $\pi_{C 2} \leq \pi<(N-M)(N-\rho M)$, the SSS equilibrium offers are $w_{H}^{*}=\bar{w}_{l}$ $+\pi\left[R_{L}(h)-R_{L}(l)\right]$ for H-firms, and $w_{L}^{*}=\bar{w}_{l}$ for L-firms. Beliefs are as in (i).

An SSS equilibrium displays wage dispersion and signaling of a high-quality job through wage premia if the high-quality worker population is large enough. The size of this wage premium depends on market conditions in the $L$-sector; it is large if each $L$-firm meets at least one $l$-worker, small otherwise. Inefficient matches occur $((1-\pi) \rho M l$-workers are matched with $H$-firms) and there is unemployment (of measure $\pi(N-\rho M)$ if $\pi N<\rho M,(1-\pi) \rho M$ if $\pi N \geq \rho M)$. The measure of inefficiency in an SSS equilibrium is

$$
C_{\text {SSS }}=(1-\pi) \rho M\left(\underline{w}_{l}-R_{H}(l)\right)+X\left(R_{H}(h)-\underline{w}_{h}\right)+Y\left(R_{L}(l)-\bar{w}_{l}\right)
$$

where $X=\pi(N-\rho M)$ if $\pi N<\rho M$ and $X=(1-\pi) \rho M$ otherwise, and $Y$ represents the potential surplus from establishing $L-l$ matches. ${ }^{18}$ As expected, the level of inefficiency in an SSS equilibrium is lower than a PN equilibrium because in the former, $H$-firms signal their type, which avoids $L-h$ matches. Thus, $C_{\mathrm{Sss}}<C_{\mathrm{PN}}$.

3.3. Pooling/Screening (PS) Equilibria. The matching game has a PS equilibrium where workers respond differently to the pooling wage offer $w_{P}^{*}$ : $h$-workers accept the offer while $l$-workers withdraw from the market. ${ }^{19}$ For $h$-workers to accept a pooling offer and risk being matched with $L$-firms, the proportion of $H$-firms must be high (stated as $\rho>\rho_{C}$ in Proposition 4). The PS equilibrium wage is determined according to demand-and-supply considerations. If total labor supply $\pi N$ at the pooling wage exceeds the demand $M$ (i.e., $\pi \geq M / N$ ), $w_{P}^{*}$ is relatively low. Otherwise $w_{P}^{*}$ is high because firms will bid up the wage until the offer hits the limit of attracting withdrawn $l$-workers. Above the locus $L L$ in Figure $3 L$-firms have no incentive to bid up the wage (condition (2)). The region of prior beliefs such that $H$-firms do not bid up that wage is given by the area to the right of $\mathrm{HH}_{1}$ locus, or $\mathrm{HH}_{2}$ locus, depending on their equilibrium profit levels (conditions in (3)). Define $w_{i}(\rho)$ through $\rho u_{i}\left(w_{i}(\rho), H\right)+(1-\rho) u_{i}\left(w_{i}(\rho), L\right)$ $-w_{i}(\rho)=\bar{u}$ as the wage that makes $i$-workers indifferent between accepting the wage $w_{i}(\rho)$ and taking their outside option, and $\rho_{C}$ through $w_{l}\left(\rho_{C}\right)=w_{h}\left(\rho_{C}\right)$.

Proposition 4. Assume $\rho>\rho_{C}$. A PS equilibrium exists where only $h$-workers accept $w_{P}^{*}=w_{l}(\rho)$ if

$$
\frac{R_{L}(l)-w_{l}(\rho)}{\left[R_{L}(h)-w_{l}(\rho)\right] N / M-\left[R_{L}(h)-R_{L}(l)\right]} \leq \pi<\frac{M}{N}
$$

\footnotetext{
${ }^{18} Y=(1-\pi) \rho M$ if $(1-\pi) N>(1-\rho) M$ and $Y=(1-\rho) M-(1-\pi)(N-\rho M)$ if $(1-\rho) M>(1-\pi)$ $N>(1-\pi)(N-\rho M)$. Otherwise $Y=0$.

${ }^{19}$ The opposite case could not happen because by (A3) $R_{H}(l)<\underline{w}_{l} \leq w_{P}^{*}$ : $H$-firms would not make an offer that only $l$-workers accept.
} 


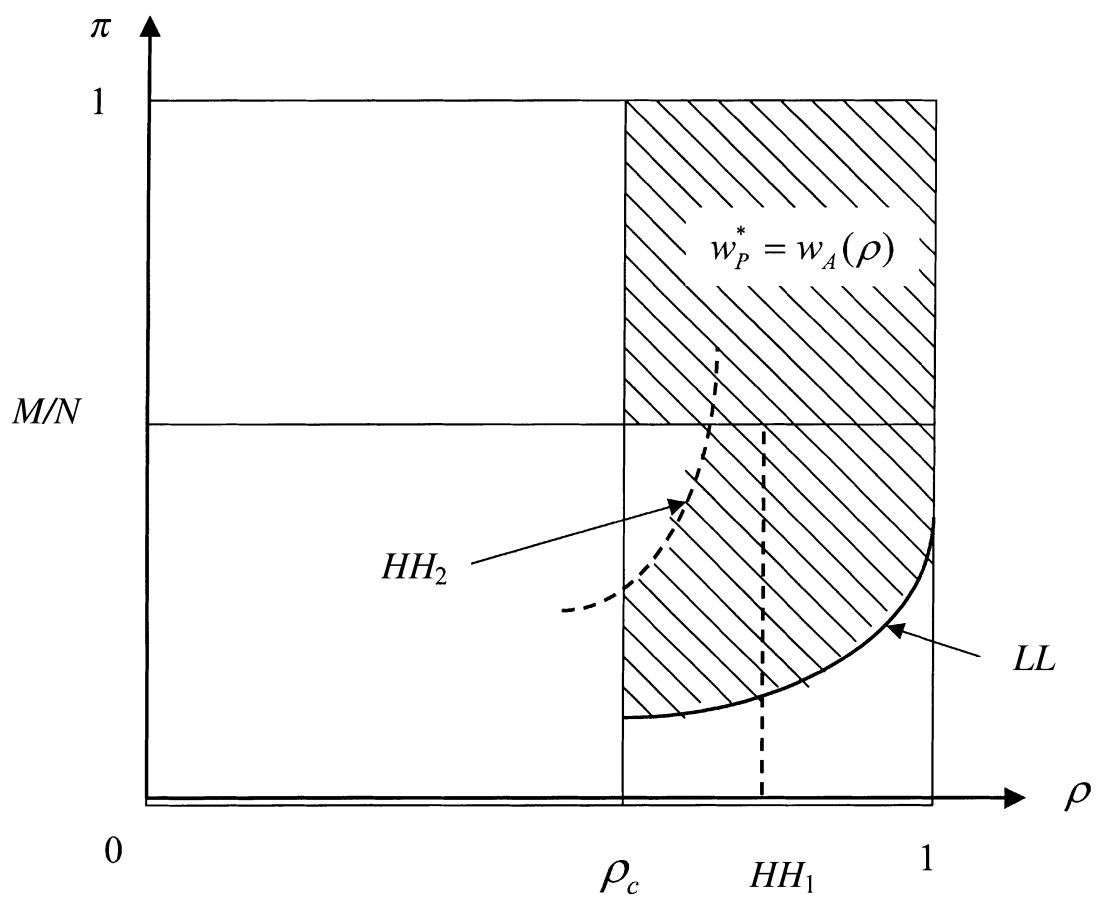

Figure 3

RANGE OF POOLING/SCREENING EQUILIBRIA AS A FUNCTION OF FIRM AND WORKER POPULATION DISTRIBUTIONS

and either

$$
\frac{M}{N} \leq \frac{R_{H}(h)-w_{l}(\rho)}{R_{H}(h)-R_{H}(l)} \quad \text { or } \quad \pi \leq \frac{w_{l}(\rho)-R_{H}(l)}{R_{H}(h)-R_{H}(l)-\frac{N}{M}\left(R_{H}(h)-w_{l}(\rho)\right)}
$$

On the other hand, if $\pi \geq M / N$, the PS equilibrium offer is $w_{P}^{*}=w_{h}(\rho)$.

This equilibrium shows that a single wage can prevail in job markets populated predominantly by high-quality firms and workers, and the equilibrium wage could be low enough to drive low-quality workers to their outside option. Though $l$-workers withdraw, they constitute a small fraction of the worker population. For $\pi N<M$, a measure $\pi \rho N$ of $H-h$ matching and a measure $(1-\rho) \pi N$ of $L-l$ matching occur. The measure of inefficiency is therefore

$$
\begin{aligned}
C_{\mathrm{PS}}= & (1-\rho) \pi N\left(\bar{w}_{h}-R_{L}(h)\right)+\left(R_{H}(h)-\underline{w}_{h}\right) \min \{\rho(M-\pi N),(1-\rho) \pi N\} \\
& +\left(R_{L}(l)-\bar{w}_{l}\right) \min \{(1-\rho) M,(1-\pi) N\}
\end{aligned}
$$

This consists of the negative surplus from $L-h$ matching, plus the foregone surpluses that could be obtained by properly matching mismatched $h$-workers and withdrawn $l$-workers.

3.4. Separating/Screening (SS) Equilibria. The last possible matching outcome involves full revelation of type information, either through strategies that 
(potentially) lead to a matching or simply through withdrawals from the market. I consider first SS equilibria in which both types of firms and workers operate, consisting of distinct wage offers $\left\{w_{L}^{*}, w_{H}^{*}\right\}$ from the two firm types. Now, since matching in an SS equilibrium occurs under perfect information, $w_{L}^{*} \geq \bar{w}_{l}$ and $w_{H}^{*} \geq \underline{w}_{h}$. $l$-Workers must reject $H$-firms' offer $\left(w_{H}^{*}<w_{L}^{*}\right)$ and firms must earn nonnegative profits: $R_{H}(h)-w_{H}^{*} \geq 0$ and $R_{L}(l)-w_{L}^{*} \geq 0$. Combining these conditions implies that $w_{L}^{*} \in\left[\bar{w}_{l}, R_{L}(l)\right]$ and $w_{H}^{*} \in\left[\underline{w}_{h}, w_{L}^{*}\right]$. Note that an $H$-firm never imitates the higher wage offer of an $L$-firm. An imitation in the opposite direction is possible, but ruled out under the conditions given in Proposition 5. Note that perfect matching is obtained; hence an SS equilibrium is efficient.

As shown in Figure 4, an SS equilibrium exists if $\pi$ is sufficiently lower than $\rho$ and if $M / N$ is close to one (stated as (4) in Proposition 5). The relatively low wage offered by $H$-firms is not imitated by an $L$-firm thanks to a low probability of meeting an $h$-worker, which implies a low $\pi / \rho$ ratio. This, combined with a ratio $M / N$ sufficiently close to one, implies that $l$-worker population exceeds $L$-firm population; hence, $L$-firms meet an $l$-worker with probability one. Note that the SS equilibrium produces the efficient outcome.

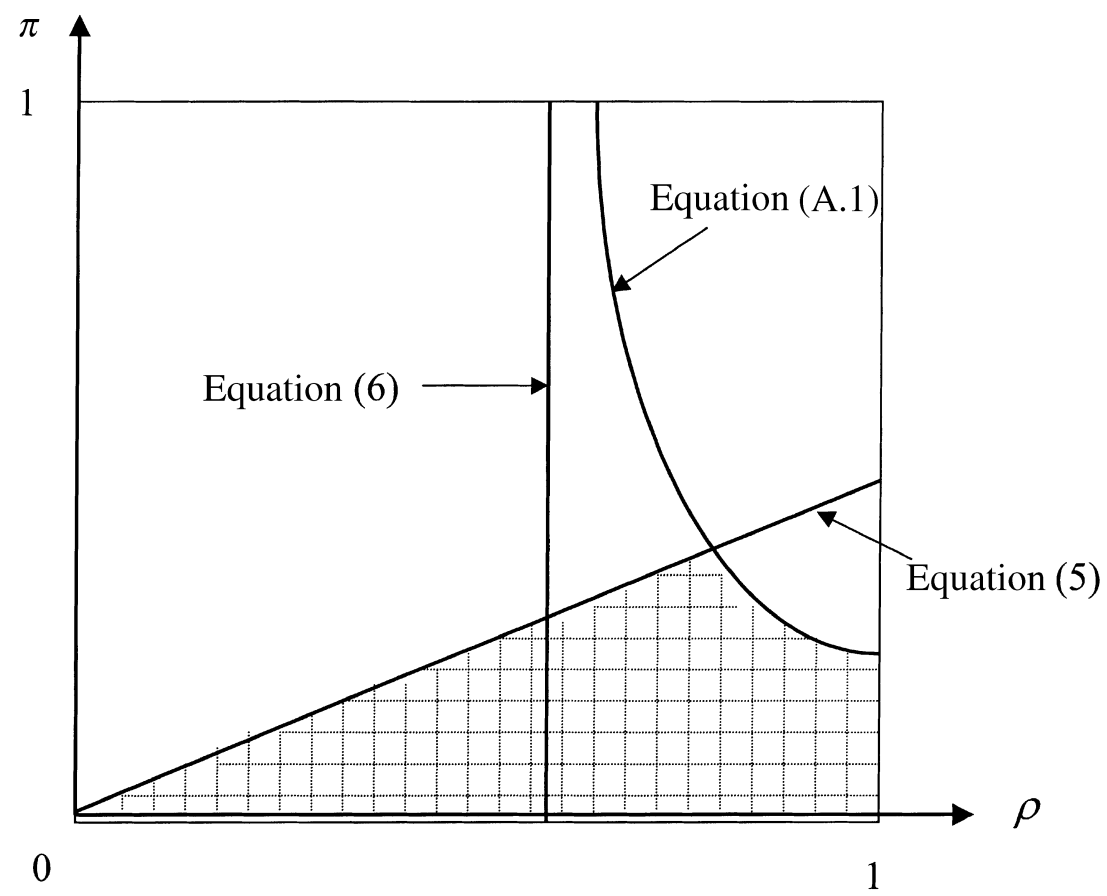

FIGURE 4 
Proposition 5. If

$$
\pi \leq \frac{\rho M}{N}\left(\frac{R_{L}(l)-\bar{w}_{l}}{R_{L}(h)-\underline{w}_{l}}\right)
$$

and if either

$$
\rho<\left(\frac{N}{M}\right)\left(\frac{R_{H}(h)-\underline{w}_{l}}{R_{H}(h)-R_{H}(l)}\right) \quad \text { or } \quad \pi \leq \frac{\underline{w}_{l}-R_{H}(l)}{R_{H}(h)-R_{H}(l)-(N / \rho M)\left[R_{H}(h)-\underline{w}_{l}\right]}
$$

an SS equilibrium exists where H-firms offer $w_{H}^{*}=\underline{w}_{l}$, which is accepted by h-workers, and L-firms offer $w_{L}^{*}=\bar{w}_{l}$, which only l-workers accept.

Consider now the second type of SS equilibrium where only one firm type and one worker type operate. These cannot be $H$-firms and $h$-workers because $L$-firms and/or $l$-workers would enter the market rather than withdraw. An SS equilibrium in which only $L-l$ matching occurs may exist if $l$-worker population is sufficiently large and if workers hold "pessimistic" beliefs: a wage offer is interpreted as an $L$-firm's offer unless it exceeds $R_{L}(h)$, the productivity of $h$-workers in $L$-firms. The equilibrium outcome generates Gresham's Law in the labor market: $H$-firms and $h$-workers withdraw. The wage offer of $L$-firms may take on two values, determining how the surplus from $L-l$ matching is shared. The social cost of having $H$-firms withdrawn from the market is $C_{\mathrm{SSL}}=\left(R_{H}(h)-\underline{w}_{h}\right) \min \{\rho M, \pi N\}$, which vanishes as either $\rho \rightarrow 0$ or $\pi \rightarrow 0$.

Proposition 6. Two types of SS equilibria exist where only L-firms and l-workers operate. If $\rho \leq 1-(1-\pi) N / M$ and

$$
\pi \leq \frac{R_{L}(h)-R_{H}(l)}{R_{H}(h)-R_{H}(l)}
$$

L-firms offer $w_{L}^{*}=R_{L}(l)$. If $\rho>1-(1-\pi) N / M$ and (6) holds, L-firms offer $w_{L}^{*}=$ $\bar{w}_{l}$. These offers are accepted by l-workers. H-firms do not make any offer or make a ridiculous offer rejected by all workers.

\section{SUMMARY AND DISCUSSION}

This article investigates the role wage offers can play in signaling and extracting information about unobservable firm and worker characteristics in a large job market. It considers a market matching game with two types of firms and workers: one that has desirable qualities and another with poor, undesirable qualities. Efficiency requires matching firms and workers of the same type. In the model, firms announce wages, workers make applications, and matching occurs. The article shows that when firms and workers are incompletely informed about each others' characteristics, a rich class of wage patterns and matching outcomes can arise, depending on the fraction of firms and workers with desirable attributes, the size of excess supply of labor (as captured by $N / M$ ), and matching preferences of firm and worker types. I summarize below the results and discuss their implications with reference to the literature, followed by some extensions. 
(i) Markets with a large population of "high-quality" workers have an SSS equilibrium in which wages signal firm characteristics but job applications do not signal worker types. This is the only outcome if, in addition, the population of lowquality firms is sufficiently large. Better jobs offer a higher but nonscreening wage that attracts all workers. Low-quality workers who have not been able to get a good job apply to the lower wage offered by low-quality jobs, while high-quality workers who do not find a high-quality match remain unemployed. This equilibrium outcome is supported by "pessimistic" but plausible beliefs, in that wages lower than highquality firms' equilibrium offer are interpreted as coming from low-quality firms.

The equilibrium wage offer of high-quality firms is higher than under complete information; hence, workers who match with them receive a "wage premium." The theoretical literature provides several explanations for why profit-maximizing firms would pay wages above opportunity costs of workers. Weiss' (1990) explanation is based on the premise that higher wages per se increase output. He shows that the matching market populated by identical firms and heterogeneous workers with unobserved qualities and reservation wages has a complete sorting equilibrium where higher-ability workers match with firms offering higher wages. Shirking models (e.g., Shapiro and Stiglitz, 1984) stress the fact that in many jobs it is prohibitively costly to write and enforce complete contracts that could induce efficient performance; hence, firms pay above market wages and rely on the threat of firing poorly performing workers. Firms may also be paying premia to reduce turnover (e.g., Salop, 1979) or prevent unionization (Dickens, 1986). I show that a higher wage may be used as a signal of desirable firm characteristics when the signal is not too costly, that is, if the proportion of workers with desirable characteristics is sufficiently large.

(ii) Markets with a large proportion of high-quality firms to low-quality workers have an SS equilibrium where wages signal firm quality and screen worker types. Perfect matching occurs despite the information problem. The wage structure is the opposite of (i), hence accords with the prediction of the theory of compensating differences: less attractive jobs pay a premium. This outcome arises here from signaling and screening considerations, rather than through self-selection of heterogeneous but informed workers who weigh wages against job attributes. Compensating differentials may be paid even if the differences in question are not observable but have to be experienced.

(iii) If the low-quality worker population is sufficiently large, high-quality firms may withdraw from the market. The intuition is straightforward: "lemons" dominate the market and drive high-quality firms and workers to their outside options. Note that the range of parameters (preponderance of low-quality types) generating this outcome in part intersects with the outcome in (ii).

(iv) Three types of equilibria coexist in a market dominated by high-quality firms and workers. The first is the SSS equilibrium described in (i). Second, there is a PN equilibrium in which strategies are devoid of type information: all firms offer the same wage, accepted by all workers. Thus, a single wage prevails in this market populated by observationally identical but heterogeneous firms and workers. The third is a PS equilibrium in which all firms offer the same wage, accepted only by high-quality workers. The likelihood of this outcome decreases as worker and firm 
populations become equal in size. The PS equilibrium differs from PN in that poorquality workers (who constitute a small fraction of the worker population) withdraw from the market. ${ }^{20}$ Both equilibria are supported by plausible beliefs. The main intuition behind a pooling wage offer is that signaling desirable job attributes is not worth the cost given workers' beliefs and preponderance of high-quality workers. ${ }^{21}$

I close the article with possible extensions of the model. Introducing observable characteristics correlated with unobservables appears to have a predictable impact. The case for signaling and screening will become stronger and wage offers will become more firm- and worker-specific as the correlation increases. Though firms will be able to discriminate between cohorts of workers and vice versa, workers and firms of a given cohort will be indistinguishable; therefore, the present analysis remains relevant. ${ }^{22}$

I assumed costless search to focus exclusively on information revelation in the simplest way. Introducing a friction in the form of search costs will complicate the workers' problem. Each worker will then have to anticipate the number of applicants and trade off wages against acceptance probabilities. Introducing time dynamics is the most important and interesting extension despite the potential problem of multiple equilibria. ${ }^{23}$ In a multiperiod version of the present model, new firms and workers with unknown characteristics would join the market in each period, affecting the distribution of unattached workers and vacancies, hence the evolution of equilibrium wage offers. Wolinsky's (1990) model of information revelation through pairwise meetings is relevant here. This extension would also allow one to address important issues such as job creation and destruction.

\section{APPENDIX}

A.1. Conditions on Out-of-Equilibrium Beliefs. Formal statements of the two conditions imposed on equilibria, explained at the end of Section 2, are given below in order.

\footnotetext{
${ }^{20}$ Since they produce different matching outcomes, SSS, PN, and PS equilibria exhibit different degrees of inefficiency. An SSS equilibrium is more efficient compared to PN because it involves one less type of inefficiency, the one that stems from $H-l$ matching. The comparison between PN and PS is not that clear, however. While PS has the advantage of avoiding $H-l$ matching, it has the disadvantage of eliminating the surplus that could be generated by matching low-quality firms with withdrawn low-quality workers.

${ }^{21}$ Kuhn (1994) provides an alternative explanation for pooling contract offers, based on riskaverse and homogeneous workers' need for insurance against revelation of firm types or private information that affects workers' utilities.

${ }^{22}$ On the other hand, with more than two types of workers and firms, the number of equilibria would obviously be larger: a subset of firms may offer a pooling wage while another subset separates through different wage offers; some types of workers may have identical acceptance strategies while others signal their types or withdraw. The analysis of this general case in the present model would no doubt be considerably more complex. See Sattinger (1995) for a different approach to the matching problem with many types of workers and firms.

${ }^{23}$ In a two-period, one-sided incomplete information model, Laing (1993) studies the feedback from wages that signal worker abilities to job applications in the beginning of workers' careers. A similar effect would be observed in an equilibrium of a two-period extension of the present model.
} 
(B1) Consider a vector of equilibrium wage offers $\left\{w^{*}\right\}$ and a corresponding vector of profits $\left\{v^{*}\right\}$. For any $w_{0} \notin\left\{w^{*}\right\}$, construct the set of firm types $F\left(w_{0}\right)$ such that a firm of type $m \in F\left(w^{*}\right)$ has equilibrium profits $v_{m}^{*}$ no less than any profits it can obtain in equilibria of the continuation game following its deviation to $w_{0}$. For any firm type $j \notin F\left(w_{0}\right)$, let $\hat{\rho}_{j}=0$ if $j=L$ and $\hat{\rho}_{j}=1$ if $j=H$ given the wage offer $w_{0}$. If $v_{j}^{*}<v_{j}\left(w_{0}, \hat{\rho}_{j}\right)$, then $\left\{w^{*}\right\}$ cannot be an equilibrium vector of offers.

(B2) Let $w^{*}$ be an offer made in an equilibrium by a set $F$ of firms, with expected profits $v^{*}$ and updated beliefs $\hat{\rho}^{*}$ at $w^{*}$. Suppose that $v_{m}\left(w_{m}^{*}+\epsilon\right)>v^{*}$ for some firm $m \in F$ and $\epsilon>0$ arbitrarily small, given workers' best replies to $w^{*}+\epsilon$ with beliefs constant at $\hat{\rho}^{*}$. Then, $w^{*}$ is not an equilibrium offer.

A.2. Proof of Proposition 2. The proof first defines the set of wages that both worker types would accept, as a function of $\rho$. Next it defines the set of prior beliefs such that all firms earn nonnegative expected profits in a PN equilibrium. Last, it verifies that no deviation will occur from the prescribed strategies. I postulate beliefs for out-of-equilibrium offers as $\hat{\rho}=0$ for $w<w_{P}^{*}, \hat{\rho}=\rho$ for $w \in\left[w_{P}^{*}, R_{L}(h)\right)$ and $\hat{\rho}=1$ for $w \geq R_{L}(h)$, where $w_{P}^{*}$ is determined below. Note that $\hat{\rho}=\rho$ and $\hat{\pi}=\pi$ in a PN equilibrium because strategies do not convey any type information.

To be accepted, the pooling offer $w_{P}$ must satisfy the participation constraints of all workers:

$$
U_{i}\left(\rho, w_{P}\right) \equiv \rho u_{i}\left(w_{P}, H\right)+(1-\rho) u_{i}\left(w_{P}, L\right) \geq \bar{u}
$$

Given $\rho$, let $w_{i}(\rho)$ be the wage that makes condition (A.1) binding for at least one $i=l, h$. Also, define $w_{P}(\rho)=\max \left\{w_{l}(\rho), w_{h}(\rho)\right\}$ as the lowest wage that satisfies the participation constraints of both worker types. The assumption (A2) on the ranking of utilities, combined with (A.1), reveals that as $\rho \rightarrow 1$, $U_{h}\left(\rho, w_{P}\right)>U_{l}\left(\rho, w_{P}\right)$. Therefore, $w_{l}(\rho)>w_{h}(\rho)$; hence $w_{P}(\rho)=w_{l}(\rho)$, for $\rho$ close enough to one. Recall that by definition, $w_{l}(\rho) \rightarrow \underline{w}_{l}$ as $\rho \rightarrow 1$. On the other hand, as $\rho$ approaches zero, $U_{h}\left(\rho, w_{P}\right)<U_{l}\left(\rho, w_{P}\right)$, thus $w_{l}(\rho)<w_{h}(\rho)$, and $w_{P}(\rho)=w_{h}(\rho)$. By definition, $w_{h}(\rho) \rightarrow \bar{w}_{h}$ as $\rho \rightarrow 0$. Since $u_{i}(., j)$ is a continuous function of $w, w_{P}(\rho)$ decreases continuously in $\rho$ in the range $\left[\underline{w}_{l}, \bar{w}_{h}\right]$. We define a lower bound $\bar{\rho}$ for $\rho$ through

$$
w_{P}(\bar{\rho})=w_{h}(\bar{\rho})=\bar{w}_{l}
$$

If $\rho>\bar{\rho}, l$-workers would reject the wage $w_{P}(\rho)$ offered by $L$-firms (provided they infer the firm type) because $w_{P}(\rho)$ is lower than the minimum wage they would accept to work in a type- $L$ firm.

I construct below the set of prior beliefs $(\pi, \rho)$, denoted $S_{\mathrm{PN}}$, such that all market participants expect a nonnegative payoff given the pooling wage offer $w_{P}(\rho)$. To this end, for any $w$ and $j=L, H$, define the function $\pi_{j}(w)$ through the zero-profit condition

$$
\pi_{j}(w) R_{j}(h)+(1-\pi(w)) R_{j}(l)-w=0
$$

The function $\pi_{j}(w)$ is decreasing in $w$. The boundary of the set $S_{\mathrm{PN}}$ can be obtained by substituting for $w$ in (A.2) the lowest (pooling) wage accepted by both types, $w_{P}(\rho)$. 
To see the behavior of the functions $\pi_{H}(w)$ and $\pi_{L}(w)$, let $\rho \rightarrow 1$ and consider (A.2) for $j=H$. Using $w_{P}(1)=\underline{w}_{l}$ in (A.2) reveals that $\pi_{H}\left(w_{P}(1)\right)=\pi_{H}\left(\underline{w}_{l}\right) \in(0,1)$ (because $R_{H}(l)<\underline{w}_{l}$ but $R_{H}(h)>\underline{w}_{l}$, such a number $\pi_{H}\left(\underline{w}_{l}\right)$ strictly between zero and one must exist). Consider (A.2) for $j=L$, as $\rho \rightarrow 1$. Now, $\pi_{L}\left(w_{P}(1)\right)=\pi_{L}\left(\underline{w}_{l}\right)<0$ because from (A3) $\underline{w}_{l}<\bar{w}_{l}<R_{L}(l)<R_{L}(h)$. On the other hand, as $\rho$ approaches zero, $w_{P}(\rho)$ approaches $w_{P}(0)=\bar{w}_{h}$, which, used in (A.2), yields $\pi_{H}\left(\bar{w}_{h}\right)<1$ and $\pi_{L}\left(\bar{w}_{h}\right)>1$ (i.e., $\pi_{H}\left(w_{P}(0)\right)<1$ and $\left.\pi_{L}\left(w_{P}(0)\right)>1\right)$. Now define $S_{\mathrm{PN}}=\{(\pi, \rho) \mid$ $\left.(\pi, \rho) \in(0,1)^{2}, \pi \geq \pi_{j}\left(w_{P}(\rho)\right), j=L, H\right\}$.

To complete the proof, I show below that if $\pi \geq \pi_{i}\left(w_{P}\right)$ and $\rho>\bar{\rho}$, then $w_{P}^{*}=w_{P}(\rho)$ is a PN equilibrium wage offer. Workers accept the offer $w_{P}^{*}$ for it yields them a nonnegative expected surplus, by satisfying their participation constraints in (A.1). However, $N-M$ workers will be involuntarily unemployed. A slightly lower wage offer will be rejected by $h$-workers because beliefs are then revised to $\hat{\rho}=0$. $l$-Workers, too, will reject a lower offer because the condition $\rho>\bar{\rho}$ implies $w_{P}^{*}<\bar{w}_{l}$ (accepting such an offer yields a negative payoff). Since $N>M, \alpha=1$; hence the firms have no incentive to offer a higher wage. In particular, $H$-firms gain nothing by signaling their type via the high offer $w \geq R_{L}(h)$. Finally, the condition $(\pi, \rho) \in S_{\mathrm{PN}}$ ensures that all market participants obtain a nonnegative expected payoff.

A.3. Proof of Proposition 3. As a first step, I define two critical prior beliefs $\pi_{C 1}$ and $\pi_{C 2}$ as follows: If $\pi \geq \pi_{C 1}$, an $H$-firm's expected profit from making a nonscreening offer (accepted by all and ranked at top) is higher than an $L$-firm's corresponding expected profit. This condition yields

$$
\pi_{C 1}=\frac{R_{L}(l)-R_{H}(l)}{R_{L}(l)-R_{H}(l)+R_{H}(h)-R_{L}(h)}
$$

The expression defining $\pi_{C 2}$ is obtained by substituting $\bar{w}_{l}$ for $R_{L}(l)$ in the numerator of the expression defining $\pi_{C 1}$ above. The level of $\pi_{C 2}$ is such that the nonscreening wage $\bar{w}_{l}+\pi_{C 2}\left[R_{L}(h)-R_{L}(l)\right]$ yields a zero profit to $H$-firms. Note that $\pi_{C 2}<\pi_{C 1}$ because $\bar{w}_{l}<R_{L}(l) .{ }^{24}$ Out-of-equilibrium beliefs are specified as $\hat{\rho}=1$ if $w \geq w_{H}^{*}$, and $\hat{\rho}=0$ otherwise.

(i) The equilibrium offer $w_{L}^{*}=R_{L}(l)$ yields $L$-firms zero profits. $L$-firms will not deviate to $w \geq w_{H}^{*}$ because though this offer would be interpreted as coming from an $H$-firm, it can only yield nonpositive expected profits. To see that $w_{L}^{*}=R_{L}(l)$ is the only possible equilibrium offer of $L$-firms, note that the condition $\pi>(N-M) /(N-\rho M)$ ensures that $L$-firm population exceeds $l$-worker population who is not able to meet an $H$-firm, therefore, that $L-l$ matching occurs with probability less than one. Consequently, $w_{L}<R_{L}(l)$ cannot be an SSS equilibrium offer of $L$-firms because any $L$-firm would unilaterally deviate to $w_{L}+\epsilon$ and increase its probability of meeting an $l$-worker to one. As for $H$-firms, the condition $\pi \geq \pi_{C 1}$ ensures that each obtains a nonnegative expected profit, by definition of $\pi_{C 1}$. $H$-firms' offer is optimal given the belief systems because offering $w>w_{H}^{*}$ only

\footnotetext{
${ }^{24}$ This follows from Assumption (A.3), which states that the surplus from $L-l$ matching is positive.
} 
increases the wage bill leaving the expected applicant type constant, while an offer $w<w_{H}^{*}$ is interpreted as coming from an $L$-firm, hence generating negative profits. Finally, workers' application strategies are clearly optimal.

(ii) In this case, $L$-firms' equilibrium profit is $R_{L}(l)-\bar{w}_{l}>0$ and just equal to what each expects by unilaterally deviating to $H$-firms' offer $w_{H}^{*}$. The condition $\pi \leq(N-M) /(N-\rho M)$ implies that at any $w_{L} \in\left[w_{L}^{*}, R_{L}(l)\right]$ an $L$-firm meets an $l$-worker with probability one. This implies that a wage $w_{L}>\bar{w}_{l}$ cannot be an SSS equilibrium offer of $L$-firms. $H$-firms obtain nonnegative profits by condition $\pi \geq \pi_{C 2}$. As in case (i), $H$-firms' equilibrium offer is the lowest separating and nonscreening wage offer.

A.4. Proof of Proposition 4. First, define a critical belief $\rho_{C}$ through the condition $\rho_{C} u_{i}\left(w_{P}\left(\rho_{C}\right), H\right)+\left(1-\rho_{C}\right) u_{i}\left(w_{P}\left(\rho_{C}\right), L\right)=\bar{u}, i=l, h$, which implies $w_{P}\left(\rho_{C}\right)$ $=w_{l}\left(\rho_{C}\right)=w_{h}\left(\rho_{C}\right)$ (see also condition (A.1) in the proof of Proposition 2): the pooling wage $w_{P}\left(\rho_{C}\right)$ yields both worker types the expected utility $\bar{u}$. Recall that for $\rho>\rho_{C}(>\bar{\rho}), w_{h}(\rho)<w_{l}(\rho)$. Beliefs for out-of-equilibrium wage offers are specified as follows: $\hat{\rho}=\rho$ for $w<R_{L}(h)$, and $\hat{\rho}=1$ for $w \geq R_{L}(h)$.

For any $\rho>\rho_{C}, w_{P}^{*}$ must belong to the interval $\left(w_{h}(\rho), w_{l}(\rho)\right]$. Consider any $w_{P}$ from this interval. The expected profit of a type-j firm is $\alpha\left(R_{j}(h)-w_{P}\right)$ where $\alpha=1$ if $\pi N \geq M$. The wage $w_{P}^{*}=w_{h}(\rho)$ is therefore a PS equilibrium offer: a deviation to $w>w_{h}(\rho)$ only increases the wage bill (note that beliefs at the right neighborhood of $w_{P}^{*}$ satisfy (B2)), whereas a deviation to $w<w_{h}(\rho)$ is interpreted as coming from $L$-firms. Thus, $H$-firms will not deviate, nor will $L$-firms because $\rho>\rho_{C}>\bar{\rho}$ implies $w_{P}^{*}<\bar{w}_{l}$ (see the proof of Proposition 2).

Consider now the case $\pi N<M$, hence $\alpha<1$. The profit $\alpha\left(R_{j}(h)-w_{P}^{*}\right)$ is positive because $w_{P}^{*} \leq w_{l}(\rho)<\bar{w}_{l}<R_{L}(h)<R_{H}(h)$. A wage $w_{P}<w_{l}(\rho)$ cannot be a PS equilibrium offer because deviating to a wage $w_{P}+\epsilon<w_{l}(\rho)$, which by (B2) should leave beliefs unchanged, will increase $\alpha$ to one. To see that no deviation from $w_{P}^{*}=w_{l}(\rho)$ will occur given beliefs off the equilibrium path, consider first $H$-firms: deviating to a lower wage decreases profits to zero, while deviating to a $w_{P}^{*}+\epsilon$ is not beneficial if

$$
\frac{\pi N}{M}\left[R_{H}(h)-w_{l}(\rho)\right] \geq \pi R_{H}(h)+(1-\pi) R_{H}(l)-w_{l}(\rho)-\epsilon
$$

which as $\epsilon \rightarrow 0$, holds for

$$
\pi \geq \frac{R_{H}(l)-w_{l}(\rho)}{R_{H}(h)(N / M-1)+R_{H}(l)-(N / M) w_{l}(\rho)}
$$

The first condition stated in (3) implies that the denominator of the expression in (A.4) is positive; hence, the right-hand side of (A.4) becomes negative because $R_{L}(l)<w_{l}(\rho)$. If the denominator in (A.4) is negative, that is, if the first condition in (3) is false, the inequality in (A.4) must be reversed, which corresponds to the second condition given in (3). Consider now $L$-firms. As mentioned above, $\rho>\rho_{C}>\bar{\rho}$ ensures that an $L$-firm will not deviate to a lower offer. Deviating to $w_{l}(\rho)+\epsilon$, on the other hand, yields $\pi R_{L}(h)+(1-\pi) R_{L}(l)-w_{l}(\rho)-\epsilon$. This is not beneficial, because letting $\epsilon \rightarrow 0$, 


$$
\pi \geq \frac{R_{L}(l)-w_{l}(\rho)}{\left[R_{L}(h)-w_{l}(\rho)\right](N / M)-\left[R_{L}(h)-R_{L}(l)\right]}
$$

as stated in (2). Note that the belief systems are consistent with the strategies. The proof is complete.

A.5 Proof of Proposition 5. Specify out-of-equilibrium beliefs as $\hat{\rho}=1$ for $w \leq \underline{w}_{l}$ and $w \geq R_{L}(h)$, and $\hat{\rho}=0$ for $w \in\left(\underline{w}_{l}, R_{L}(h)\right)$. According to the strategies described in the proposition, an $L$-firm's equilibrium profit is $R_{L}(l)-\bar{w}_{l}$. Condition (4) ensures that $L$-firms will not deviate to any $w \leq \underline{w}_{l}$. As for out-of-equilibrium offers, an $H$-firm obtains $\pi R_{H}(h)+(1-\pi) R_{H}(l)-\underline{w}_{l}-\epsilon$ if it deviates to $\underline{w}_{l}+\epsilon$ where, by (B2), $\hat{\rho}=1$ for $\epsilon$ arbitrarily small. But this deviation merely decreases expected profits below the equilibrium profit $[(\pi N) /(\rho M)]\left[R_{H}(h)-\underline{w}_{l}\right]$ under either condition given in (5).

A.6 Proof of Proposition 6. All $L$-firms obtain zero profit in the first equilibrium. Since by the condition $\rho \leq 1-(1-\pi) N / M L$-firm population is larger than $l$-worker population, competition bids the wage up to $w_{L}^{*}=R_{L}(l)$. An offer above $R_{L}(l)$ yields negative profits whereas lower offers are rejected by all. Given beliefs $\hat{\rho}=0$ for $w<R_{L}(h)$, an $H$-firm can offer $R_{L}(h)$, signal its type, and attract all workers. However, this yields the expected profit $\pi R_{H}(h)+(1-\pi) R_{H}(l)-R_{L}(h)$, which is nonpositive because $\pi$ satisfies (6). In the second SS equilibrium, the condition $\rho>1-(1-\pi) N / M$ ensures that a unilateral deviation by an $L$-firm to a lower wage $w_{L}$ is beneficial for all $w_{L}>\bar{w}_{l}$. L-firms' equilibrium offer is therefore $w_{L}^{*}=\bar{w}_{l}$.

\section{REFERENCES}

BAC, M., "On-the-Job Specific Training and Efficient Screening," Journal of Labor Economics 18 (2000), 681-701.

Bulow, J., AND L. Summers, "A Theory of Dual Labor Markets with Application to Industrial Policy, Discrimination, and Keynesian Unemployment,' Journal of Labor Economics 4 (1986), 376-414.

CAIN, G., "The Challenge of Segmented Labor Market Theories to Orthodox Theory," Journal of Economic Literature 14 (1976), 1215-57.

Cho, I. K., AND D. KrePs, "Signaling Games and Stable Equilibria," Quarterly Journal of Economics 52 (1987), 172-221.

Coles, M. C., "Turnover Externalities with Marketplace Trading," International Economic Review 40 (1999), 851-68.

Dickens, W. T., "Wages, Employment and the Threat of Collective Action by Workers," mimeo, University of California, Berkeley, 1986.

Gibbons, R., And K. J. Murphy, “Optimal Incentive Contracts in the Presence of Career Concerns: Theory and Evidence," Journal of Political Economy 100 (1992), 468-505.

Hammond, P. J., M. KAneko, ANd M. H. Wooders, "Continuum Economies with Finite Coalitions: Core, Equilibria and Widespread Externalities," Journal of Economic Theory 49 (1989), 113-34.

Holtzer, H. J., L. F. KAtz, AND A. B. Krueger, “Job Queues and Wages,” Quarterly Journal of Economics 106 (1991), 739-68.

KuHn, P., "Nonrevelation in Employment Contracts," International Economic Review 35 (1994), 261-82. 
LAING, D., “A Signaling Theory of Nominal Wage Rigidity,” Economic Journal 103 (1993), 1493510.

LAzEAR, E. P., “Why is There Mandatory Retirement?,” Journal of Political Economy 87 (1979), 1261-84.

Leonard, J. S., “Executive Pay and Firm Performance," Industrial and Labor Relations Review 43 (1990), S13-S29.

Masters, A. M., "Wage Posting in Two-Sided Search and the Minimum Wage," International Economic Review 40 (1999), 809-26.

Rosen, S., "The Theory of Equalizing Differences," in O. Ashenfelter and R. Layard, eds., Handbook of Labor Economics, Vol. 1 (New York: Elsevier Science, 1986).

RoY, A. D., "Some Thoughts on the Distribution of Earnings," Oxford Economic Papers 3 (1951), 135-46.

Salop, S. C., "A Model of the Natural Rate of Unemployment," American Economic Review 69 (1979), 117-25.

SATtinger, M., "Search and the Efficient Assignment of Workers to Jobs," International Economic Review 36 (1995), 283-98.

Shapiro, C., And J. E. Stiglitz, "Equilibrium Unemployment as a Worker Discipline Device," American Economic Review 74 (1984), 433-44.

Thaler, R. H., “Anomalies: Interindustry Wage Differentials,” Journal of Economic Perspectives 3 (1989), 181-93.

WeIss, A., Efficiency Wages (Oxford: Clarendon Press, 1990).

Wolinsky, A., "Information Revelation in a Market with Pairwise Meetings," Econometrica 58 (1990), 1-23. 
